\title{
Remineralizing Agent -Then and Now -An Update
}

Naveena Preethi $\mathbf{P}^{*}$, Nagarathana $\mathrm{C}$ and Sakunthala BK

Department of Pedodontics and Preventive Dentistry, Rajarajeswari Dental College and Hospital, Kumbalgodu, Mysore Road, Bangalore, India

*Corresponding author: Naveena Preethi P,Postgraduate Student, Department of Pedodontics and Preventive Dentistry, Rajarajeswari Dental College and Hospital, Kumbalgodu, Mysore Road, Bangalore -560074, India, Tel: +919844066427; E-mail: naveenapreethi@gmail.com

Rec Date: Sept 03, 2014; Acc Date: Oct 20, 2014; Pub Date: Oct 27, 2014

Copyright: (C) 2014 Naveena P, et al., This is an open-access article distributed under the terms of the Creative Commons Attribution License, which permits unrestricted use, distribution, and reproduction in any medium, provided the original author and source are credited.

\begin{abstract}
Dental caries is a highly prevalent multifactorial disease and has been a major public health problem for many centuries. The goal of modern dentistry is to manage non-cavitated caries lesions non-invasively through remineralization in an attempt to prevent disease progression and improve aesthetics, strength, and function. Remineralization is defined as the process whereby calcium and phosphate ions are supplied from an externalsource to the tooth thereby converting ion deposition into crystal voids in demineralized enamel, thus producing net mineral gain. Biomimetic approaches to stabilization of bioavailable calcium, phosphate, and fluoride ions and the localization of these ions to non-cavitated caries lesions for controlled remineralization shows great promise for the non-invasive management of dental caries. The aim of this article is to give a brief update about current remineralization agent aiming to "treat early caries lesion" noninvasively.
\end{abstract}

Keywords: Early carious lesion; Non-invasive treatment; Remineralizing agent

\section{Introduction}

Worldwide contribution of dental caries to the burden of oral diseases is about 10 times higher than that of periodontal disease, than other common oral conditions [1]. Owing to its globally high prevalence, dental caries is a 'pandemic' disease characterized by a high percentage of untreated carious cavities causing pain, discomfort and functional limitations [2]. Untreated carious cavities, furthermore, has significant impact on the general health of children and on social and economic wellbeing of communities [3].

A surgical approach for the elimination of carious lesion was developed only a century ago; this approach was necessary during that time, because there was no valid alternative. The focus on caries has recently shifted to the development of methodologies for the detection of the early stages of caries lesions and the use of non-invasive treatment for these lesions. The non-invasive treatment of early carious lesions by remineralization has the potential to be a major advance in the clinical management of the disease.

Remineralization of white-spot lesions and carious lesions may be possible with a variety of currently available agents containing fluoride, bioavailable calcium and phosphate, and casein phosphopeptide in-amorphous calcium phosphate, self-assembling peptide. The current concept further bridges the traditional gap between prevention, non-invasive and surgical procedures which is just what dentistry needs for the current age.

\section{Demineralization/Remineralization}

Demineralization occurs by disassociation of lactic acid, produced by bacterial carbohydrate metabolism, with tooth mineral. This reaction leads to release of mineral ions into the solution.

$$
\mathrm{Ca}_{10}\left(\mathrm{PO}_{4}\right)_{6}(\mathrm{OH})_{2}+14 \mathrm{H}^{+} \rightarrow 10 \mathrm{Ca}^{+}+6 \mathrm{H}_{2} \mathrm{PO}_{4}+\mathrm{H}_{2} \mathrm{O}
$$

The extent to which tooth mineral dissolves in a given solution is governed by thermodynamic ion activityproduct (IAP).

$$
\text { IAP }=\left(\mathrm{Ca}^{2+}\right)_{10}\left(\mathrm{PO}_{4}^{3}\right)_{6}(\mathrm{OH})_{2}
$$

When the IAP equals a constant called the solubility product constant of $\mathrm{K}_{\mathrm{sp}}$, the solution is in equilibriumwith the solid and is said to be saturated with respect to the solid [4]. The only requirement for dimeralization to occur is that the IAP in the demineralizing solution should be less than the $\mathrm{K}_{\mathrm{sp}}$.

$$
\left(\mathrm{Ca}^{2+}\right)_{10}\left(\mathrm{PO}^{3}\right)_{6}\left(\mathrm{OH}^{-}\right)_{2}<\mathrm{K}_{\mathrm{sp}} \text { (tooth mineral) }
$$

The subsurface lesion is reversible via a remineralization process. This is by the increase in oral fluid calcium and phosphate that drives the remineralizationprocess.

Enamel demineralization in the presence of (F) Fluoride in dental biofilm: Sugars (sucrose, glucose, fructose) are converted to acids in the biofilm. When the $\mathrm{pH}$ decreases to below 5.5, undersaturation happens with respect to hydroxyapatite (HA) and then reaches the biofilm fluid, resulting in mineral dissolution. However, if the $\mathrm{pH}$ is higher than 4.5 and $\mathrm{F}$ is present,the biofilm fluid is supersaturated with respect to fluorapatite (FA) and there is reprecipitation of minerals in the enamel. As a consequence, the net demineralization is reduced [5] (Figure 1). 
Page 2 of 5

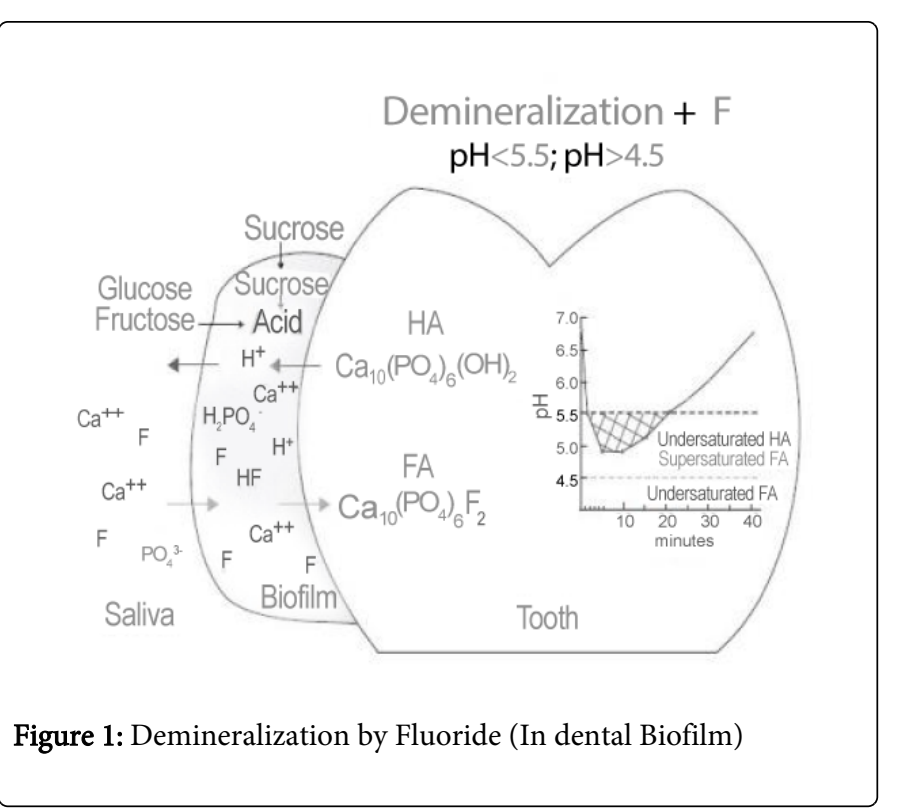

Enamel remineralization in the presence of $\mathrm{F}$ in dental biofilm. After the exposure to sugars has ceased, acids in the biofilm are cleared by saliva and converted to salts. As a result, the $\mathrm{pH}$ increases and, at 5.5 or higher, the biofilm fluid is supersaturated with respect to HA and FA. If the biofilm still contains F, then thelost $\mathrm{Ca}$ and Pby enamel can be recovered more efficiently [5] (Figure 2).

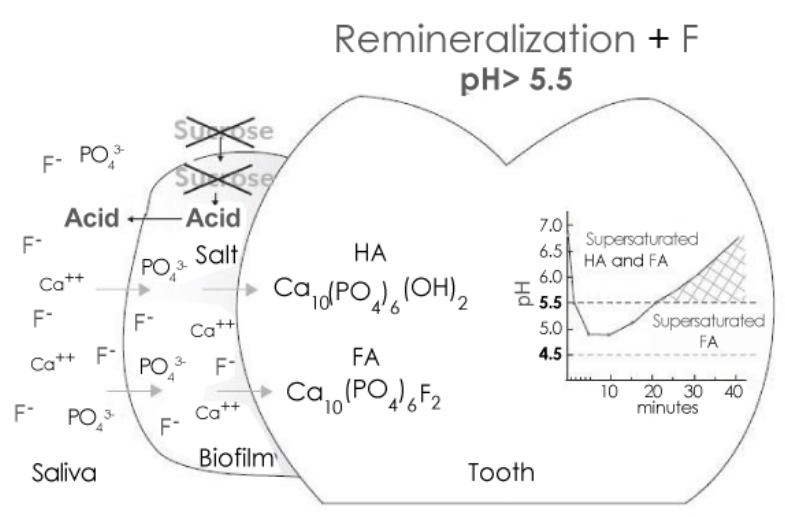

Figure 2: Remineralization by Fluoride (In dental Biofilm)

\section{Ideal requirements of a remineralizing agent}

- Should deliver calcium and phosphate into the subsurface

- Should not deliver any excess of calcium

- Should not favor calculus formation

- Should work at an acidic pH so as to stop demineralization during a carious attack

- Should work in xerostomic patients also, as saliva cannot effectively stop the

carious process
- Should be able to boost the remineralizing properties of saliva

- The novel materials should be able to show some benefits over fluoride [6].

\section{Indication}

An adjunct to preventive therapy for reducing caries in high-risk patients

- Reduce dental erosion in patients with gastric reflux or other disorders

- To reduce decalcification in orthodontic patients

- To repair enamel in cases involving whitespot lesions

- For fluorosis, before and after teeth whitening and to desensitize sensitive teeth

\section{[7].}

\section{Past remineralizing agent-fluorides}

The first set of theories concerning the mechanism of action of fluoride was based exclusively on its pre-eruptive effect. Arnold, in 1957, was the first author to mention the post-eruptive effect of fluoride in the drinking water and the ability of topical fluoride to reduce the incidence of caries [4-8].

The mechanism by which fluoride increases caries resistance may arise from both systemic and topical applications of fluoride and can be broadly grouped as follows - increased enamel resistance, increased rate of maturation, remineralization of incipient caries, interference with micro-organisms and improved tooth morphology [8].

\section{Casein Phosphopeptide - Amorphous Calcium Phosphate}

The casein phosphopeptides (CPPs) are produced from the tryptic digest of casein, aggregated with calcium phosphate and purified through ultrafiltration [6]. Casein has the ability to stabilize calcium and phosphate ions by releasing small sequences of peptides (CPPs) through partial enzymic digestion that leads to the development of a remineralization technology based on casein phosphopeptidestabilized amorphous calcium phosphate complexes (CPP-ACP) and casein phosphopeptide-stabilized amorphous calcium fluoride phosphate complexes (CPP -ACFP) $[9,10]$.

This technology was developed by Eric Reynolds, Australia. CPPs contain the cluster sequence of -Ser (P)-Ser (P)- Ser (P)- Glu-Glu from casein $[11,12]$. This protein nanotechnology combines the precise ratio of 144 calcium ions plus 96 phosphate ions and six peptides of CPP.

The size and electro neutrality of the CPP nanocomplexes allows them to diffuse down the concentration gradient into the body of the sub-surface lesion $[9,10]$. Once present in the enamel sub-surface lesion, the CPP-ACP releases the weakly bound calcium and phosphate ions $[13,14]$, depositing them into crystal voids. The CPPs have a high binding affinity for apatite [15] thus, on entering the lesion, the CPPs binds to the more thermodynamically favored surface of an apatite crystal face.

It is $\mathrm{pH}$ responsive, i.e. with increasing $\mathrm{pH}$, the level of bound $\mathrm{ACP}$ increases, stabilizing free calcium and phosphate and thus provides an anti-calculus action [16]. The anti-caries action influences the properties and behavior of dental plaque through (1) binding with 
adhesion molecules in mutans Streptococci, impairing their incorporation into plaque, (2) elevating plaque calcium ion levels to inhibit plaque fermentation and (3) providing protein and phosphate buffering of plaque fluid $\mathrm{pH}$ to suppress overgrowth of aciduric species when fermentable carbohydrate is in excess.

Tooth crèmes using CPP-ACP (Recaldent technology) such as MIPaste and ToothMousse16 recognizes the importance of the neutral ion species, gaining access to the sub-surface lesion through porous enamel surface. This is the reason why arrested white spot lesions should have a surface etching treatment before remineralization with Recaldent products, unlike fluoride treatments with conventional dentifrices $(1000 \mathrm{ppm})$ that deposits surface mineral but do not eliminate a white-spot lesion [17].

CPP-ACP is a useful cario-static agent for the control of dental caries [7]. A dentifrice containing CPP-ACP with fluoride will provide remineralization, which is superior to both CPP-ACP and to conventional and high fluoride dentifrices [18]. Studies done by Reynolds and colleagues also found a reduction of $15 \%$ and $46 \%$, respectively, in $0.1 \%$ and $1.0 \% \mathrm{w} / \mathrm{v}$ CPP-ACP [19].

Thus, it is evident that other than fluoride, the strongest clinical evidence for remineralization is CPP-based Recaldent technology, with both long-term, large-scale clinical trials and randomized controlled clinical trials further supporting its efficacy.

\section{Bioactive glass}

Bioactive glass (Bioglass ${ }^{\circ}$ ) was invented by Dr. Larry Hench in1960s. It acts as a biomimetic mineralizer matching the body's own mineralizing traits and also affecting cell signals thereby benefitting the restoration of tissue structure and function [20].

Bioglass ${ }^{\circledast}$ in an aqueous environment immediately begins surface reaction in three phases-leaching and exchange of cations, network dissolution of $\mathrm{SiO}_{2}$ and precipitation of calcium and phosphate forming an apatite layer. The critical stages for glass surface reactions are the initial $\mathrm{Na}^{+}$and $\mathrm{H}^{+} / \mathrm{H}_{3} \mathrm{O}^{+}$ion exchange and de-alkalinization of the glass surface layer which is quite rapid, within minutes of implantation and exposure to body fluids [21]. The net negative charge on the surface and loss of sodium causes localized breakdown of the silica network with the resultant formation of (silanol) Si (OH) groups, which then repolymerizes into a silica-rich surface layer [22]. Within 3-6 h in vitro, the calcium phosphate layer will crystallize into the carbonated hydroxyl apatite (CAP) layer, which essentially is the bonding layer. Chemically and structurally, this apatite is nearly identical to bone and tooth mineral. These Bioglass ${ }^{\oplus}$ surface reactions from implantation to formation of 100-150 $\mu \mathrm{m}$ CAP layer takes 12-24 h [21-23].

Bioactive glass formulation commonly used in research studies contains 45 wt $\% \mathrm{SiO}_{2} 4.5 \mathrm{wt} \% \mathrm{Na}_{2} \mathrm{O}$ and $\mathrm{CaO}$ and $6 \mathrm{wt} \% \mathrm{P}_{2} \mathrm{O}_{5}$. The network breakdown of silica depends upon the concentration of $\mathrm{SiO}_{2}$ and is time dependent. Thus, keeping the silica below $60 \mathrm{wt} \%$ and maintaining a high $\mathrm{CaO} / \mathrm{P}_{2} \mathrm{O}_{5}$ ratio guarantees a highly reactive surface.

Novamin $^{\oplus}$, a trade name for bioactive glass, is manufactured by Novamin Technologies Inc. (Alachua, FL, USA). It has been demonstrated that, fine particulate bioactive glasses $(<90 \mu \mathrm{m})$ incorporated into an aqueous dentifrice has the ability to clinically reduce the tooth hypersensitivity through the occlusion of dentinal tubules by the formation of the CAP layer [24]. Investigators using bioactive glass compositions have demonstrated a significant antimicrobial effect toward caries pathogens (S. mutans, S. sanguis) upon exposure to bioactive glass powders as well as solutions and extracts [25].

Caries can also result from inadequate saliva, without which fluoride is limited by value [26]. Thus, individuals who experience reduced calcium, phosphate and fluoride ions caused by hyposalivation can benefit from the use of bioactive glass. In addition, women are at increased caries risk due to inadequate salivary calcium levels at different points in their lives, including ovulation, pregnancy and post-menopause, resulting in the same net effect as reduced saliva fluoride efficacy.

Thus, the use of bioactive glass (Novamin Technology) in remineralization of enamel is quite promising, especially in patients with systemic problems, but further research needs to be undertaken to prove its efficacy.

\section{Tri-calcium phosphate [clinprotooth crème]}

TCP is a new hybrid material created with a milling technique that fuses beta tricalcium phosphate ( $(\mathrm{-TCP})$ and sodium lauryl sulfate or fumaric acid. This blending results in "functionalized" calcium and a "free" phosphate, designed to increase the efficacy of fluoride remineralization $[27,28]$. $\beta-$ TCP is similar to apatite structure and possesses unique calcium environments capable of reacting with fluoride and enamel. When the phosphate floats freely, the exposed calcium environments are protected by preventing the calcium from prematurely interacting with fluoride. TCP provides catalytic amounts of calcium to boost fluoride efficacy and may be well designed to coexist with fluoride in a mouth rinse or dentifrice because it will not react before reaching the tooth surface [29]. When TCP finally comes into contact with the tooth surface and is moistened by saliva, the protective barrier breaks down, making the calcium, phosphate and fluoride ions available to the teeth. The fluoride and calcium then reacts with weakened enamel to provide a seed for enhanced mineral growth relative to fluoride alone.

Products available with TCP include a 5000 ppm sodium fluoride dentifrice and a $5 \%$ sodium fluoride varnish. Studies have concluded that TCP provided superior surface and sub-surface remineralization compared with a $5000 \mathrm{ppm}$ fluoride and CPP-ACP [30]. There has been no significant research on TCP combined with fluoride varnish. All published studies supporting this material has been invitro studies. The potential of TCP is promising, but more studies are needed, including clinical trials supporting its efficacy in boosting remineralization.

\section{ACP Technology [enamelon, enamel care]}

The ACP technology requires a two-phase delivery system to keep the calcium and phosphorous components from reacting with each other before use. The current sources of calcium and phosphorous are two salts, calcium sulfate and dipotassium phosphate. When the two salts are mixed, they rapidly form ACP that can precipitate over the tooth surface. This precipitated ACP can then readily dissolve into the saliva and can be available for tooth remineralization [31].

The ACP technology was developed by Dr. Ming S. Tung. In 1999, ACP was incorporated into toothpaste called Enamelonand later reintroduced in 2004 as Enamel Care toothpaste [32].There is modest evidence for Enamelon ${ }^{\text {tw }}$ on caries inhibitory action [33]. 
An inherent technical issue with Enamelon ${ }^{\mathrm{m}}$ is that calcium and phosphate are not stabilized, thus allows the two ions to combine into insoluble precipitates before they come into contact with saliva or enamel. This is unlike Recaldent ${ }^{\mathrm{m}}$, which has the casein phosphoproteins to stabilize calcium and phosphate.

\section{Self assembling peptide}

Recent developments in tissue engineering, material sciences and stem cell research offer considerable potential to dental therapies. Peptide treatment for early caries lesion is the area of current research.

Peptide treatment significantly increases net mineral gain due to a combined effect of increased mineral gain and inhibition of mineral loss. Rationally designed $\beta$-sheet-forming peptides P114 that selfassemble themselves to form three-dimensional scaffolds under defined environmental conditions have been shown to nucleate hydroxyl apatite de novo and to have potential applications in mineralized tissue regeneration, mimicking the action of enamel matrix proteins during tooth development. Results suggest that a single application of P114 can be beneficial in the treatment of early caries lesions and that self assembling peptides are candidate materials for mineralized tissue regeneration and repair [34].

Anionic P114 is a rationally-designed self-assembling peptide $[35,36]$. Self-assembling peptides undergo well-characterized hierarchial self-assembly as three-dimensional fibrillar scaffolds in response to specific environmental triggers, offering a new generation of well-defined biopolymers with a range of potential applications. At certain peptide concentrations P114 switches from a low viscosity isotropic liquid to an elastomeric nematic gel $(\mathrm{pH}<7.4)$ then the anionic groups of the P114 side chains would attract $\mathrm{Ca}++$ ions, inducing de novo precipitation of hydroxyl apatite inducing mineral deposition in situ [37].

P114 is safe, non-invasive and acceptable to patients. The treatment differs from other 'filling without drilling'. The use of a biomimetic peptide such as P114 has the additional advantage of effecting 'natural' repair by regenerating the mineral itself. P114 is a well-tolerated treatment, and currently designed to test 'next generation' peptides to accelerate the repair process, thus making 'filling without drilling' a reality [37].

In a recent study by Schlee et al. also proved that when P114 is applied to the tooth the peptide diffuses into the subsurface microproes and forms a $3 \mathrm{D}$ scaffold which is made up of small fibers these scaffold mimies proteins found in teeth development and supports hydroxyl apatite cryteststallisation around it to regenerate tooth enamel over a period of three months [38], another invitro study which evaluated the efficacy of the newly introduced self-assembling peptide P114 (curodontTM) for regenerating demineralized tooth tissue on smooth surface also showed significant results based on test and control group after 8 and 12 weeks [39].

\section{Conclusion}

The relationship by which remineralization could occur more favorably can be understood, only by clearly knowing the mode of implementing these remineralizing agents. Thus it is important for dental professionals to be aware of these techniques and know, that it takes significant time to establish the bonafides of a new technology like this. And further requires more detailed clinical knowledge and trials along with a "watching brief" methodology necessary for this rapidly progressing area of dental science.

\section{References}

1. Aggeli A, Bell M, Boden N, Keen JN, Knowles PF, et al. (1997) Responsive gels formed by the spontaneous self-assembly of peptides into polymeric beta-sheet tapes. Nature 386: 259-262

2. Aggeli A, Bell M, Boden N (1997) Engineering of peptide beta-sheet nanotapes. J Mater Chem; 7: 1135-1145

3. Al-Khateeb S, Exterkate R, Angmar-Månsson B, Ten Cate JM (2000) Effect of acid-etching on remineralization of enamel white spot lesions. Acta Odontol Scand 58: 31-36

4. Allan I, Newman H, Wilson M (2001) Antibacterial activity of particulate bioglass against supra- and subgingival bacteria. Biomaterials 22: 1683-1687

5. Azarpazhooh A, Limeback H (2008) Clinical efficacy of casein derivatives: a systematic review of the literature.J Am Dent Assoc 139: 915-924

6. Brunton PA, Davies RP, Burke JL, Smith A, Aggeli A, et al. (2013) Treatment of early caries lesions using biomimetic self-assembling peptides--a clinical safety trial. Br Dent J 215: E6

7. Cross KJ, Huq NL, Palamara JE, Perich JW, Reynolds EC (2005) Physicochemical characterization of casein phosphopeptide-amorphous calcium phosphate nanocomplexes. J BiolChem 280: 15362-15369

8. Cross KJ, Huq NL, O’Brien-Simpson NM, Perich JW, Attard TJ, et al. (2007) The role of multiphosphorylated peptides in mineralized tissue regeneration. Int J Pept Res Ther; 13: 479-495

9. Cochrane NJ, Saranathan S, Cai F, Cross KJ, Reynolds EC (2008) Enamel subsurface lesion remineralisation with casein phosphopeptide stabilized solutions of calcium, phosphate and fluoride. Caries Res; 42: 88-97

10. Cury JA, Tenuta LM (2009) Enamel remineralization: controlling the caries disease or treating early caries lesions? Braz Oral Res 23 Suppl 1: 23-30

11. Cochrane NJ, Reynolds EC (2009) Casein phosphopeptides in oral health. In: Food constituents and oral health: Current status and future prospects. In: Wilson M, Editor. Cambridge: CRC Press.

12. Edelstein BL (2006) The dental caries pandemic and disparities problem. BMC Oral Health 6 Suppl 1: S2

13. Greenspan DC (1999) Developments in biocompatible glass compositions. Medical Device and Diagnostic Industry Magazine MDDI Article index. An MD and DI, column special section; Mar: 150

14. Hench LL, Wilson J (1993) An introduction to bioceramics. World Scientific Publishing, Singapore

15. Jablonski-Momeni A, Heinzel-Gutenbrunner M (2014) Efficacy of the self-assembling peptide P11-4 in constructing a remineralization scaffold on artificially-induced enamel lesions on smooth surfaces. J Orofac Orthop 75: 175-190

16. Jensen ME, Wefel JS (1989) Human plaque pH responses to meals and the effects of chewing gum. Br Dent J 167: 204-208

17. Kontonasaki E, Zorba T, Papdopoulou L, Pavlidou E, Chatzistavrou X, et al. (2002) Hydroxycarbonate apatite formation on particulate biolgass in vitro as a function of time. Cryst Res Technol 37: 1165-1171

18. Kirkham J, Firth A, Vernals D, Boden N, Robinson C, et al. (2007) Selfassembling peptide scaffolds promote enamel remineralization. J Dent Res 86: 426-430

19. Karlinsey RL, Mackey AC (2009) Solid state preparation and dental application of an organically modified calcium phosphate. J Mater Sci 44: 34-69

20. Karlinsey RL, Mackey AC, Walker ER, Frederick KE (2010) Surfactantmodified beta-TCP: structure, properties, and in vitro remineralization of subsurface enamel lesions. J Mater Sci Mater Med 21: 2009-2020

21. Karlinsey RL, Mackey AC, Walker ER, Frederick KE (2010) Preparation, characterization and in vitro efficacy of an acid-modified beta-TCP 
Citation: Naveena P, Nagarathana C, Sakunthala BK (2014) Remineralizing Agent -Then and Now -An Update. Dentistry 4: 256. doi: $10.4172 / 2157-7633.1000256$

Page 5 of 5

material for dental hard-tissue remineralization. Acta Biomater 6 969-978

22. Karlinsey RL, Mackey AC, Walker ER, Amaechi BT, Karthikeyan R, et al. (2010) Remineralization potential of 5000 ppm fluoride dentifrices evaluated in a pH cycling model. J Dent Oral Hyg 2: 1-6

23. Litkowski LJ, Hack GD, Sheaffer HB, Greenspan DC (1997) Occlusion of dentin tubules by 45 S5Bioglass ${ }^{\oplus}$. Bioceramics ; 10

24. Leach SA, Lee GT, EdgarWM (1989) Remineralization of artificial carieslike lesions in human enamel in situ by chewing sorbitol gum. J Dent Res 68: $1064-1068$

25. Leone CW, Oppenheim FG (2001) Physical and chemical aspects of saliva as indicators of risk for dental caries in humans. J Dent Educ 65: 1054-106.

26. Mellberg RJ, Ripa WL, Leske SG (1983) Fluoride in preventive dentistry Theory and clinical applications. Quintessence Publishing Co., Inc. Chicago.

27. Murray CJL, Lopez AD (1996) The global burden of disease. A comprehensive assessment of mortality and disability from diseases injuries, and risk factors in 1980 and projected to 2020. Cambridge, Mass: Harvard University Press

28. Reynolds EC (1998) Anticariogenic complexes of amorphous calcium phosphate stabilized by casein phosphopeptides: a review. Spec Care Dentist 18: 8-16.

29. Reynolds EC (2008) Calcium phosphate-based remineralization systems scientific evidence? Aust Dent J 53: 268-273
30. Reynolds EC, Cai F, Cochrane NJ, Shen P, Walker GD, et al. (2008) Fluoride and casein phosphopeptide-amorphous calcium phosphate. J Dent Res 87: 344-348

31. Schlee M, Rathe F (2013) Effect of Curodont Repair in patients with proximal carious lesions: uncontrolled, non-interventional study interim report. Clinical Oral Investigations 17: 1046-1047

32. Sullivan RJ, Charig A, Blake-Haskins J, Zhang YP, Miller SM, et al. (1997) In vivo detection of calcium from dicalcium phosphate dihydrate dentifrices in demineralized human enamel and plaque. Adv Dent Res 11: 380-387

33. Sheiham A (2006) Dental caries affects body weight, growth and quality of life in pre-school children. Br Dent J 201: 625-626

34. Thompson A, Grant LP, Tanzer JM (1999) Model for assessment of carious lesion remineralization, and remineralization by a novel toothpaste. J Clin Dent 10: 34-39

35. Tung MS, Eichmiller FC (1999) Dental applications of amorphous calcium phosphates. J Clin Dent 10: 1-6

36. Tyagi SP, Garg P, Sinha DJ, Singh UP (2013) An update on rematerializing agents. J Interdiscip Dentistry 3: 151-158

37. Walsh LJ (2007) Tooth Mouse: Anthology of applications. GCAsiaPte Ltd, Singapore.

38. Walsh LJ (2009) Contemporary technologies for remineralization therapies: A review. Int Dent SA 11: 6-16

39. Zero DT (2006) Dentifrices, mouthwashes, and remineralization/caries arrestment strategies. BMC Oral Health 6 Suppl 1: S9 